\title{
Crystallization of two forms of a cyclodextrin inclusion complex containing a common organic guest
}

\author{
Mino R. Caira,* Elise J. C. de Vries and Luigi R. Nassimbeni \\ Department of Chemistry, University of Cape Town, Rondebosch 7701, South Africa. \\ E-mail: xraymino@science.uct.ac.za; Fax: +27 21689 7499; Tel: +27 216503071
}

Received (in Cambridge, UK) 22nd May 2003, Accepted 20th June 2003

First published as an Advance Article on the web 9th July 2003

The isolation and structural elucidation by single crystal Xray diffraction of triclinic and monoclinic modifications of an inclusion complex of $\beta$-cyclodextrin with the same guest, methylparaben, are reported.

The alkyl esters of 4-hydroxybenzoic acid ('alkylparabens', $\mathrm{HO}-\mathrm{C}_{6} \mathrm{H}_{4}-\mathrm{CO}-\mathrm{OR}, \mathrm{R}=\mathrm{Me}, \mathrm{Et}, n$-Pr, $n$-Bu) are widely used as preservatives in pharmaceutical formulations, food products and cosmetics. ${ }^{1}$ Despite their simplicity, they display some interesting properties including anomalous aqueous solubilities when mixtures of the homologues containing ethyl- and propylparaben are investigated. This behaviour has been explained on the basis of solid solution formation between these crystallographically isostructural components. ${ }^{2}$ The intrinsically low aqueous solubilities of the alkylparabens has also prompted investigation of their ability to complex with synthetic and semisynthetic cyclodextrins as a route for solubilisation. ${ }^{3-5}$ Proof of the formation of inclusion complexes between alkylparabens and $\beta$-cyclodextrin based on thermogravimetry, differential scanning calorimetry and X-ray powder diffraction has been reported. ${ }^{6}$

The present report concerns our most recent finding, namely that reaction between methylparaben and $\beta$-cyclodextrin leads to the formation of two distinct crystalline inclusion complexes, depending on the preparative method employed. $\dagger$ A survey of available cyclodextrin crystal structures ${ }^{7}$ revealed that this is the first instance of structurally different inclusion complexes of a cyclodextrin with a common organic guest. The possibility of generating different crystalline forms of a cyclodextrin inclusion complex by systematic variation of crystallization conditions has been alluded to previously. ${ }^{8}$ This has now been realized and we report the successful X-ray analyses of two modifications of the $\beta$-cyclodextrin inclusion complex of methylparaben which crystallize in the space groups $P 1$ (triclinic, complex 1) and $C 2$ (monoclinic, complex 2) $\ddagger$ and discuss the general significance of this result.

In both crystals the host $\beta$-cyclodextrin forms the wellknown, stable dimeric motif via $\mathrm{O}-\mathrm{H} \cdots \mathrm{O}$ hydrogen bonding between the secondary faces of the two components of the dimer ${ }^{9}$ thus providing a large cavity which accommodates the guest molecules. Fig. 1 shows stereoscopic views of the dimeric complex units in the triclinic and monoclinic structures. For the triclinic complex, the entire dimer shown in Fig. 1 is crystallographically unique whereas only one half of the dimer of the monoclinic form is unique, the two halves being related by a crystallographic diad. The latter runs horizontally in Fig. 1, passing through the dimer interface.

Both complexes have a $1: 1$ host-guest stoichiometric ratio, as determined by UV spectrophotometry and elemental analysis. Location and crystallographic modelling of the guests were rendered difficult due to their disorder in both inclusion complexes. In complex 1, two pairs of independent guest conformers were eventually located and refined with variable site-occupancy factors (s.o.f.'s) constrained to sum to unity for each pair. This led to s.o.f.'s of $\sim 0.75$ for the major components and $\sim 0.25$ for the minor components. In complex $\mathbf{2}$, each methylparaben molecule was found to be likewise disordered over two positions but with similar electron densities for the disordered components and each was therefore modelled with s.o.f. 0.5. Guest phenyl rings were modelled as regular hexagons and several distance and angle restraints were imposed to ensure reasonable geometries for the ester moieties. The mode of guest inclusion is analogous in the two complexes, the phenyl rings occupying central positions within their respective host molecules, the guest hydroxy groups being located at the primary rims and the ester groups occupying the dimeric interfaces. Intersection of the phenyl rings of the disordered components is a common feature in these complexes, indicating that the central region of each host favours interaction with this hydrophobic moiety. In complex 1 the angles of intersection are significantly larger than those in complex 2 . The nature of this disorder is similar to that found for the guest paracetamol [ $N$-(4-hydroxyphenyl)acetamide] in its dimeric inclusion complex with $\beta$-cyclodextrin ${ }^{10}$ which crystallizes in the space group $C 2$ and whose host dimer is isostructural with that in complex 2. A further similarity between the paracetamol complex and those reported here is the location of the guest hydroxy group at the primary rims of the host molecules. We attribute this invariant guest orientation to the stabilisation afforded by hydrogen bonding between the guest hydroxy group and other 'extra-cavity' hydrophilic centres. In complex 1 this hydrogen bonding involves water molecules which bridge the guest hydroxy group and host primary hydroxy groups, whereas in $\mathbf{2}$ there is direct hydrogen bonding between host and guest. In the case of the paracetamol complex, 10 the guest hydroxy group is directly hydrogen bonded to its counterpart in a symmetry-related complex unit. Solvation of polar groups has long been recognized as an
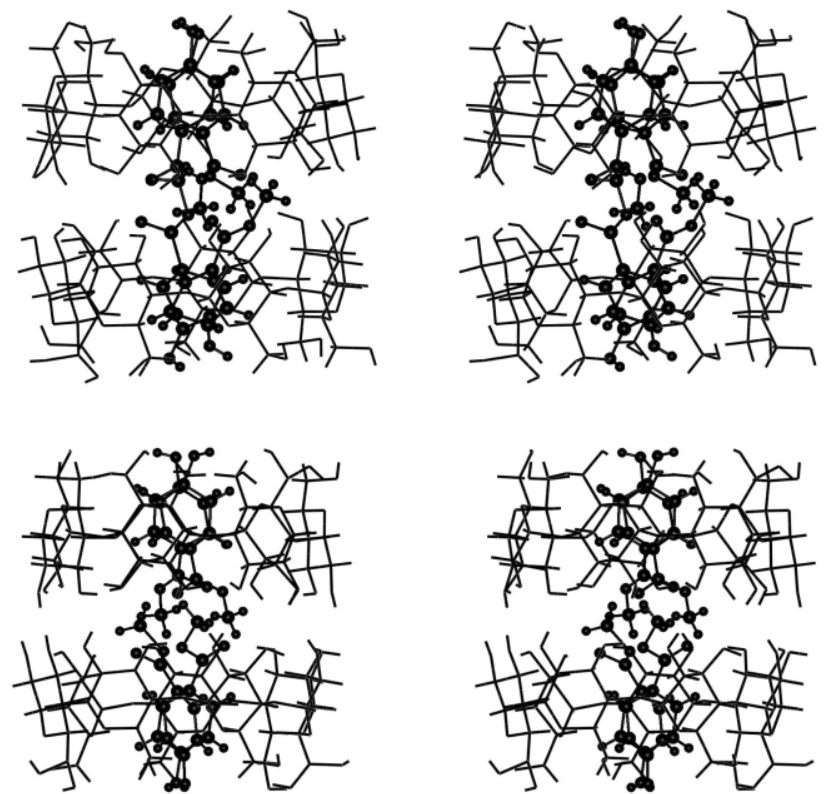

Fig. 1 Stereoscopic views of the dimeric motifs in the triclinic (upper) and monoclinic (lower) inclusion complexes of $\beta$-cyclodextrin-methylparaben. 
important factor determining the orientation of the guest in the $\beta$-cyclodextrin cavity. ${ }^{11}$

The crystals of both complexes are colourless at ambient temperature and have considerably different degrees of hydration. They show significantly different behaviour on thermal analysis, complex $\mathbf{1}$ being more stable than $\mathbf{2}$. Crystals of $\mathbf{1}$ begin to dehydrate only at $39{ }^{\circ} \mathrm{C}$ on the hot stage, turning brown at $251{ }^{\circ} \mathrm{C}$ whereas crystals of complex 2 develop cracks and dehydrate on removal of their mother liquor at room temperature and discolour at $194{ }^{\circ} \mathrm{C}$. This behaviour can be reconciled with their crystal structures as explained below. Fig. 2 shows the X-ray powder patterns of complexes $\mathbf{1}$ and $\mathbf{2}$ computed from the refined single crystal X-ray structures. The uniqueness of the patterns attests to the presence of two distinct crystal forms.

The complexes $\mathbf{1}$ and $\mathbf{2}$ belong to two known isostructural series of dimeric $\beta$-cyclodextrin complexes (12 and 11 respectively) for which 'reference' powder X-ray patterns have recently been published as an aid to confirming inclusion complex formation and identifying complex space groups. ${ }^{8}$ The dimeric complex units in $\mathbf{1}$ pack in the so-called IM ("intermediate') mode, ${ }^{9}$ characterised by close-packed layers of dimers which stack with a significant lateral shift, resulting in discontinuous channels. Water molecules are encaged within the interstices surrounding the dimers. In contrast, the dimeric units in complex 2 pack in the so-called $\mathrm{CH}$ (channel) mode, ${ }^{9}$ characterised by linear channels produced when the closepacked layers of dimers stack in exact alignment. In this arrangement, water molecules also occupy linear channels which thus provide a more facile route for dehydration, explaining the relative instability of this crystal form.

We attribute the formation of $\mathbf{1}$ and $\mathbf{2}$ to the different temperatures of crystallization employed. It was also noted that upon kneading the host with methylparaben at room temperature, the monoclinic phase $\mathbf{2}$ was invariably produced. However, recrystallization of this material from water followed by refrigeration at $7{ }^{\circ} \mathrm{C}$ yielded the triclinic form $\mathbf{1}$.

In summary, we have established the conditions for isolating different crystal forms of a cyclodextrin inclusion complex with a common organic guest and have elucidated their structures. This is the first documented case of the crystallization of different forms of a cyclodextrin inclusion complex obtained by varying the crystal growth conditions and it indicates that inclusion compounds in this class may show much richer solid state chemistry than hitherto envisaged. In addition, the finding that such crystalline modifications can display different properties (e.g. different degrees of hydration and different thermal

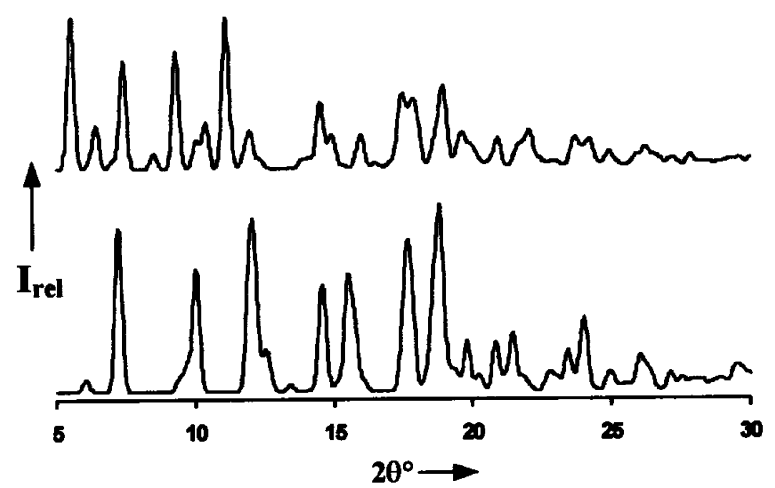

Fig. 2 Computer-generated powder X-ray patterns for the triclinic (upper) and monoclinic (lower) inclusion complexes of $\beta$-cyclodextrin-methylparaben. stabilities) is of considerable significance in the pharmaceutical industry where each solid form of a given drug substance is a new molecular entity with potential for incorporation into a solid dosage form. A full account of the interaction between the alkylparabens and $\beta$-cyclodextrin both in solution and in the solid state is in preparation. ${ }^{12}$

We thank the University of Cape Town and the National Research Foundation (Pretoria) for financial support. This material is based upon work supported by the NRF under Grant number NRF 2053361. Any opinions, findings and conclusions or recommendations expressed in the material are those of the authors and do not necessarily reflect the views of the NRF.

\section{Notes and references}

$\dagger$ Methylparaben was added to a hot $\left(45^{\circ} \mathrm{C}\right)$ aqueous solution of the host (host-guest ratio $1: 1$ ). After stirring for $2 \mathrm{~h}$, the hot solution was filtered, cooled to room temperature and stored in a refrigerator at $7{ }^{\circ} \mathrm{C}$. Crystals of complex 1 were obtained after 1 month. The same initial procedure was used to prepare complex 2 , but the solution was allowed to evaporate slowly at room temperature $\left(20^{\circ} \mathrm{C}\right)$ yielding crystals after $1-2$ weeks.

$\ddagger$ Intensity data were collected on a Nonius Kappa CCD diffractometer using graphite-monochromated Mo-K $\alpha$ X-rays $(\lambda=0.71069 \AA)$. Both crystals were coated in Paratone $\mathrm{N}$ oil (Exxon Chemical Co., TX, USA) immediately after removal from their mother liquor to prevent dehydration. Lowering the temperature of the crystal of $\mathbf{1}$ in a stream of nitrogen vapour from ambient temperature $(294 \mathrm{~K})$ to $173(2) \mathrm{K}$ for intensity data collection did not induce any phase change. Crystal data for $\mathbf{1}:\left(\mathrm{C}_{42} \mathrm{H}_{70} \mathrm{O}_{35}\right)_{2} \cdot\left(\mathrm{C}_{8}\right.$ $\left.\mathrm{H}_{8} \mathrm{O}_{3}\right)_{2} \cdot 28 \mathrm{H}_{2} \mathrm{O}, M=3078.80$, triclinic, space group $P 1, a=18.0187(3), b$ $=15.3431(4), c=15.4140(3) \AA, \alpha=103.464(1), \beta=113.122(1), \gamma=$ $99.254^{\circ}, U=3656.65(1) \AA^{3}, Z=1, D_{\mathrm{c}}=1.398 \mathrm{~g} \mathrm{~cm}^{-3}, T=173(2) \mathrm{K}$, $\mu(\mathrm{Mo}-\mathrm{K} \alpha)=0.128 \mathrm{~mm}^{-1} .18382$ unique reflections were collected and the refinement was based on all data excluding 5 low-angle reflections truncated by the beamstop. The final $w R 2=0.267$ (all data), $R 1$ [16651 data with $\left.F^{2}>2 \sigma\left(F^{2}\right)\right]=0.0937$, and goodness-of-fit on $F^{2}=1.068$ (CCDC 211386). Crystal data for 2: $\mathrm{C}_{42} \mathrm{H}_{70} \mathrm{O}_{35} \cdot \mathrm{C}_{8} \mathrm{H}_{8} \mathrm{O}_{3} \cdot 7.2 \mathrm{H}_{2} \mathrm{O}, M=1416.80$, monoclinic, space group $C 2, a=18.8632(4), b=24.4542(5), c=$ $15.5942(5) \AA, \beta=110.668(1), U=6730.4(3) \AA^{3}, Z=4, D_{\mathrm{c}}=1.398 \mathrm{~g}$ $\mathrm{cm}^{-3}, T=293(2) \mathrm{K}, \mu(\mathrm{Mo}-\mathrm{K} \alpha)=0.125 \mathrm{~mm}^{-1}$. Of the 21970 reflections collected, 14557 were unique $\left(R_{\text {int }}=0.0200\right)$. Refinement was based on all data excluding 15 low-angle reflections truncated by the beamstop. The final $w R 2=0.285$ (all data), $R 1$ [14557 data with $\left.F^{2}>2 \sigma\left(F^{2}\right)\right]=0.1002$ and goodness-of-fit on $F^{2}=1.068$ (CCDC 211387). See http:// www.rsc.org/suppdata/cc/b3/b305784b/ for crystallographic data in CIF or other electronic format.

1 Handbook of Pharmaceutical Excipients, 2nd edn., ed. A. Wade and P. J. Weller, American Pharmaceutical Association, Washington DC, 1994, p. 49.

2 F. Giordano, R. Bettini, C. Donini, A. Gazzaniga, M. R. Caira, G. G. Z Zhang and D. J. W. Grant, J. Pharm. Sci., 1999, 88, 1210.

3 J. Cohen and J. L. Lach, J. Pharm. Sci., 1963, 52, 132.

4 J. L. Lach and J. Cohen, J. Pharm. Sci., 1963, 52, 137.

5 H. Matsuda, K. Ito, Y. Sato, D. Yoshizawa, M. Tanaka, A. Taki, H. Sumiyoshi, T. Utsuki, F. Hirayama and K. Uekama, Chem. Pharm. Bull., 1993, 41, 1448 .

6 M. R. Caira, E. J. C. de Vries and L. R. Nassimbeni, J. Therm. Anal. Calorim., 2003, in press.

7 Cambridge Structural Database and Cambridge Structural Database System, Version 5.24, November 2002, Cambridge Crystallographic Data Centre, University Chemical Laboratory, Cambridge, UK.

8 M. R. Caira, Rev. Roum. Chim., 2001, 46, 371.

9 D. Mentzafos, I. M. Mavridis, G. le Bas and G. Tsoucaris, Acta Crystallogr., Sect. B. (Struct. Sci.), 1991, 47, 746.

10 M. R. Caira and D. R. Dodds, J. Incl. Phenom. Macrocyclic Chem., 2000, 38, 75

11 R. Bergeron, M. Channing, K. McGovern and W. Roberts, Bioorg. Chem., 1979, 8, 263.

12 E. J. C. de Vries, M. R. Caira, L. R. Nassimbeni, M. Bogdan, S. I. Farcas and D. Bogdan, in preparation. 